\title{
Platelet Testing is Associated with Worse Clinical Outcomes for Patients Treated with the Pipeline Embolization Device
}

\author{
W. Brinjikji, G. Lanzino, H.J. Cloft, A.H. Siddiqui, R.A. Hanel, and D.F. Kallmes
}

\begin{abstract}
BACKGROUND AND PURPOSE: The necessity for platelet-inhibition testing before aneurysm treatment in patients premedicated with antiplatelet agents is controversial. Using the International Retrospective Study of Pipeline Embolization Device registry, we studied complication rates in groups of patients who underwent platelet testing and those who did not undergo platelet testing to determine if these test results were associated with improved outcomes.
\end{abstract}

MATERIALS AND METHODS: Patients in the International Retrospective Study of Pipeline Embolization Device registry with an unruptured aneurysm were categorized as those who underwent platelet testing before Pipeline embolization device treatment or those who did not. Complication rates were compared by using the Fisher exact or Pearson $\chi^{2}$ test. Multivariate analysis was performed to determine if platelet function testing was independently associated with poor outcomes after adjusting for age, number of devices and aneurysms, aneurysm location and size, and practitioner and center volume.

RESULTS: Compared with the patients who received a Pipeline embolization device without platelet testing, those who underwent platelet testing and Pipeline embolization device placement experienced higher rates of intracranial hemorrhage (0 of 187 [0.0\%] vs 12 of 511 [2.3\%], respectively; $P=.04$ ), neurologic morbidity (4 of $187[2.1 \%]$ vs 42 of $511[8.2 \%]$, respectively; $P<.01$ ), and combined neurologic morbidity and mortality (6 of $187[3.2 \%]$ vs 45 of $511[8.8 \%$ ], respectively; $P=.01$ ). More patients in the platelet testing and Pipeline embolization device group were treated with multiple devices (227 [38.0\%] vs 56 [27.8] patients, respectively; $P=.01)$. On multivariate analysis, the group of patients who underwent platelet testing and Pipeline embolization device placement had higher odds of neurologic morbidity (OR, $3.25[95 \% \mathrm{Cl}, 1.10-9.61] ; P=.03)$.

CONCLUSIONS: Platelet testing in patients who undergo Pipeline embolization device placement is associated with higher rates of morbidity. Additional prospective studies are needed to determine if and when platelet testing in these patients is appropriate.

ABBREVIATION: PED = Pipeline embolization device

$\mathbf{T}$ he Pipeline embolization device (PED; Covidien, Irvine, California) is increasingly used in the treatment of intracranial aneurysms. ${ }^{1-4}$ The PED flow diverter is a bare-metal construct that serves as a scaffold for neointimal proliferation. ${ }^{5,6}$ Because of the thrombogenic nature of the bare-metal component of the device, dual-antiplatelet therapy is required in both the preoperative and postoperative settings, and patients are required to take dual-antiplatelet therapy for several months after the procedure.

In addition to aspirin, clopidogrel is currently the most commonly prescribed antiplatelet drug for dual-antiplatelet therapy

Received January 29, 2015; accepted after revision March 24.

From the Departments of Radiology (W.B., G.L., H.J.C., D.F.K.) and Neurosurgery (G.L., H.J.C., D.F.K.), Mayo Clinic, Rochester, Minnesota; Department of Neurosurgery (A.H.S.), State University of New York, Buffalo, New York; and Department of Neurosurgery (R.A.H.), Baptist Neurological Institute, Jacksonville, Florida.

Please address correspondence to W. Brinjikji, MD, Mayo Clinic, 200 First St SW, OL1-112 SMH, Rochester, MN 55905; e-mail: kallmes.david@mayo.edu; @wbrinjikji

http://dx.doi.org/10.3174/ajnr.A4411

among patients who undergo PED placement. However, there exists wide variability in the activation of clopidogrel among individual patients. ${ }^{7}$ As a result, platelet function testing is widely used among neurointerventionists to ensure proper function of the drug. ${ }^{8,9}$ However, controversy exists as to whether platelet testing is necessary in patients who undergo PED placement, because the benefits have yet to be proved. ${ }^{10}$ By using the International Retrospective Study of Pipeline Embolization Device (IntrePED) registry, ${ }^{11}$ we compared the clinical outcomes of patients who underwent platelet testing and those who did not to determine whether this testing was associated with better outcomes among patients who undergo PED placement.

\section{MATERIALS AND METHODS \\ Study Design and Participants}

We retrospectively evaluated all patients with an unruptured intracranial aneurysm who were treated with the Pipeline embolization device between July 2008 and February 2013 in 1 of 17 
centers in 6 countries experienced in PED use. The local institutional review boards or ethics committees approved the study and granted waivers of informed consent for use of the patients' retrospective data. This postmarket observational registry was funded and supported by Covidien, which had scientific oversight of the study's steering committee members.

Patients were included if they underwent PED treatment for an intracranial aneurysm after the date of regulatory approval in that region or country. Patients were excluded if they had not undergone "meaningful" follow-up, defined as imaging and clinical evaluations after treatment during the window of time defined by each institutional review board/ethics committee approval. Seven hundred ninety-three patients with 906 aneurysms (76 [8.4\%] ruptured, 824 [91\%] unruptured, and 6 [0.7\%] unknown) were included. Of the patients with an unruptured aneurysm, information on whether antecedent platelet testing was performed was available for 698 patients with 802 aneurysms. Any patient with a ruptured aneurysm was excluded from the analysis.

\section{Procedures}

Because this was a retrospective study, procedural details and periprocedural patient management varied across the centers. All the centers used a common study protocol. The steering committee defined neurologic "clinical safety events of interest" a priori, including spontaneous rupture of the target aneurysm causing subarachnoid hemorrhage or cavernous-carotid fistula, intraparenchymal hemorrhage, ischemic stroke, parent artery stenosis, and permanent cranial neuropathy. Site investigators identified events of interest according to the study protocol. All events of interest were reviewed in detail by an adverse events review committee, comprising 3 members of the steering committee, including the overall principal investigator. The adverse events review committee was independent of the sponsor. The committee determined the category of event and whether the event was major or minor. A major adverse event was defined as ongoing clinical deficit 7 days after the event. All major adverse events are included in the neurologic morbidity and mortality rates. The timing of every adverse event was recorded in relation to the timing of the PED procedure, not the timing of platelet testing. Information collected during the study included baseline characteristics of the patients and aneurysms, procedural information, and follow-up clinic visits or telephone calls. The use of platelet testing was indicated on the case report forms for each patient. Every patient who underwent platelet testing did so before the procedure.

\section{Baseline Characteristics and Outcomes}

Patients were categorized as those who underwent platelet testing before PED treatment (platelet testing/PED group) or those who underwent PED treatment only (PED alone group). No patient in the PED alone group underwent platelet testing, and every patient in the platelet testing/PED group underwent platelet testing before treatment. The decisions to perform platelet testing varied according to operator and center. The following baseline characteristics were compared between the groups: age, aneurysm size (in millimeters), aneurysm location (ICA, MCA, posterior nonbasilar, basilar, or other), number of PEDs used, aneurysm shape (dissecting, fusiform, saccular, or other), mean and median center volume of PED procedures during the study period, and the mean and median practitioner volume of PED procedures during the study period.

The primary outcome of this study was combined neurologic morbidity and neurologic mortality. The secondary outcomes were spontaneous rupture, intracranial hemorrhage, ischemic stroke, parent artery stenosis, cranial neuropathy, neurologic morbidity, neurologic mortality, and all-cause mortality. These outcomes were compared between the groups.

\section{Statistical Analysis}

Statistical analyses were performed by using SAS version 9.1 or higher (SAS Institute, Cary, North Carolina). Summary statistics are presented for all data available by using means and standard deviations for continuous variables and frequency tabulations for categoric variables. Comparisons between the groups for continuous variables were evaluated by using $t$ tests or ANOVA and for binary categoric variables using the Fisher exact or Pearson $\chi^{2}$ test. Most statistical analyses were performed across patient groups (ie, on a per-patient basis). Because some patients had $>1$ aneurysm, however, each patient's first aneurysm treated was used to classify him or her into the 4 anatomic/size subgroups, and the largest aneurysm was used to classify patients into 1 of the 3 aneurysm size categories. The first aneurysm treated was defined a priori.

A multivariate logistic regression analysis was performed to determine if platelet function testing was independently associated with the outcomes listed above. Adjusted variables in this model included age, aneurysm size, aneurysm location, number of aneurysms treated, number of PEDs, center volume (modeled as a continuous variable), and practitioner volume (modeled as a continuous variable). These variables were included in the model because they were associated with adverse outcomes in either the original International Retrospective Study of Pipeline Embolization Device study or subsequent subgroup analyses that are currently being performed. For complications with a low incidence rate (eg, spontaneous rupture, cranial neuropathy), the Firth penalized maximum-likelihood estimation was used to reduce bias in the parameter estimates caused by separability, as often occurs when the event is rare. Goodness-of-fit tests were performed for the multivariate models by using the Hosmer-Lemeshow models.

\section{Role of the Funding Source}

An academic principal investigator and an academic steering committee supervised the trial design and operations. The principal investigator, steering committee, and adverse events committee were independent of the sponsor. The steering committee interpreted the results, and the principal investigator wrote the report. The study sponsor was responsible for site management, data management, statistical analysis, and safety reporting. The corresponding author had full access to all study data and had final responsibility for the decision to submit for publication.

AJNR Am J Neuroradiol 36:2090-95 Nov 2015 www.ajnr.org 
Table 1: Anatomic and clinical characteristics

\begin{tabular}{|c|c|c|c|}
\hline Demographic Characteristic & $\begin{array}{c}\text { Platelet } \\
\text { Testing/PED } \\
\text { Group }\end{array}$ & $\begin{array}{l}\text { PED Alone } \\
\text { Group }\end{array}$ & $P$ Value \\
\hline No. $(\%)$ of patients & $511(73.2)$ & $187(26.8)$ & \\
\hline \multicolumn{4}{|l|}{ Age, y } \\
\hline Mean $\pm S D$ & $58.1 \pm 14.1$ & $55.7 \pm 13.2$ & .022 \\
\hline Median (minimum, maximum) & $59(9,86)$ & $57(13,81)$ & \\
\hline No. (\%) male & $98(19.2)$ & $40(21.4)$ & .52 \\
\hline \multicolumn{4}{|l|}{ Follow-up duration, mo } \\
\hline Mean $\pm S D$ & $21.5 \pm 8.0$ & $23.5 \pm 10.1$ & .20 \\
\hline Median (minimum, maximum) & $20.8(0.1,48.0)$ & $21.3(0.2,60.5)$ & \\
\hline Total no. (\%) of aneurysms & $601(74.8)$ & $201(25.2)$ & \\
\hline \multicolumn{4}{|l|}{ No. (\%) of patients with } \\
\hline 1 aneurysm & $438(85.7)$ & $176(94.1)$ & \\
\hline 2 aneurysms & $61(11.9)$ & $9(4.8)$ & .008 \\
\hline$\geq 3$ aneurysms & $12(2.4)$ & $2(1.1)$ & \\
\hline Mean center volume $\pm \mathrm{SD}, n$ & $65 \pm 45$ & $63 \pm 32$ & .06 \\
\hline Median center volume (minimum, maximum), $n$ & $47(21,149)$ & $69(19,149)$ & \\
\hline Mean practitioner volume $\pm \mathrm{SD}, n$ & $25 \pm 15$ & $21 \pm 13$ & .004 \\
\hline Median practitioner volume (minimum, maximum), $n$ & $21(1,54)$ & $21(1,54)$ & \\
\hline \multicolumn{4}{|l|}{ Aneurysm size, $\mathrm{mm}$} \\
\hline Mean $\pm \mathrm{SD}(n)$ & $10.8 \pm 7.7(595)$ & $12.0 \pm 7.7(200)$ & .01 \\
\hline Median (minimum, maximum) & $8.8(1.0,55.0)$ & $10.5(1.0,40.0)$ & \\
\hline \multicolumn{4}{|l|}{ Aneurysm location, \% $(n / N)$} \\
\hline Internal carotid artery & $78.0(469 / 601)$ & $77.1(155 / 201)$ & \\
\hline Middle cerebral artery & $4.7(28 / 601)$ & $3.0(6 / 201)$ & \\
\hline Posterior cerebral artery & $1.7(10 / 601)$ & $1.0(2 / 201)$ & .11 \\
\hline Basilar artery & $4.0(24 / 601)$ & $8.5(17 / 201)$ & \\
\hline Other & $11.6(70 / 601)$ & $10.4(21 / 201)$ & \\
\hline Multiple PEDs used & $38.0(227 / 598)$ & $27.9(56 / 201)$ & .01 \\
\hline \multicolumn{4}{|l|}{ Aneurysm shape, \% $(n / N)$} \\
\hline Saccular & 76.9 (462/601) & $77.1(155 / 201)$ & \\
\hline Fusiform & $13.1(79 / 601)$ & 13.4 (27/201) & .58 \\
\hline Dissecting & $5.0(30 / 601)$ & $6.5(13 / 201)$ & \\
\hline Other & $5.0(30 / 601)$ & $3.0(6 / 201)$ & \\
\hline
\end{tabular}

More patients in the platelet testing/ PED group were treated with multiple PEDs (227 [38.0\%] vs 56 [27.9\%] patients, respectively; $P=.01)$. No differences in aneurysm shape $(P=.58)$ or location $(P=.11)$ were seen between the groups. More patients in the platelet testing/PED group were treated for multiple aneurysms than in the PED alone group ( $14.3 \%$ vs $5.9 \%$, respectively; $P=$ .008). The median follow-up time was 19.3 months. Patients in the platelet testing/PED group had a higher mean practitioner volume than those in the PED alone group ( 25 vs 21 , respectively; $P=$ $.004)$. The mean center volumes were similar in both groups (65 [platelet testing/PED] vs 63 [PED alone]; $P=.06$ ). Ninety percent of the patients were followed up for $>12$ months. These data are summarized in Table 1.

\section{Complication Rates According to Platelet-Testing Status}

Bivariate Analysis. Among patients in the platelet testing/PED group, 12 of 511 $(2.3 \%)$ patients suffered intracranial hemorrhage compared with 0 of 187 $(0.0 \%)$ patients in the PED alone group $(P=.04)$. There was a trend toward higher rates of ischemic stroke in the platelet testing/PED group; 28 (5.5\%)

\section{Table 2: Complications}

\begin{tabular}{|c|c|c|c|}
\hline Complication & $\begin{array}{c}\text { Platelet } \\
\text { Testing/PED } \\
\text { Group } \\
\text { (N=511) } \\
\text { (\% [n]) }\end{array}$ & $\begin{array}{l}\text { PED Alone } \\
\text { Group } \\
(N=187) \\
(\%[n])\end{array}$ & $P$ Value \\
\hline $\begin{array}{l}\text { Primary outcome: neurologic } \\
\text { morbidity and neurologic } \\
\text { mortality }\end{array}$ & $8.8(45)$ & $3.2(6)$ & .01 \\
\hline \multicolumn{4}{|l|}{ Secondary outcomes } \\
\hline Spontaneous rupture & $0.6(3)$ & $0.5(1)$ & 1 \\
\hline Intracranial hemorrhage & $2.3(12)$ & $0.0(0)$ & .04 \\
\hline Ischemic stroke & $5.5(28)$ & $2.1(4)$ & .07 \\
\hline Parent artery stenosis & $0.2(1)$ & $0.5(1)$ & .46 \\
\hline Cranial neuropathy & $0.4(2)$ & $0.0(0)$ & 1 \\
\hline Neurologic morbidity & $8.2(42)$ & $2.1(4)$ & $<.01$ \\
\hline Neurologic mortality & $3.5(18)$ & $1.1(2)$ & .12 \\
\hline All-cause mortality & $4.1(21)$ & $1.1(2)$ & .05 \\
\hline
\end{tabular}

\section{RESULTS}

\section{Baseline Characteristics}

A total of 698 patients with 802 treated unruptured aneurysms were included in this study; 511 (73.2\%) patients with 601 aneurysms underwent platelet testing before PED placement, and 187 (26.8\%) patients with 201 aneurysms underwent PED placement without platelet testing. Aneurysm sizes were significantly larger in the PED alone group than in the platelet testing/PED group (mean $\pm \mathrm{SD}, 12.0 \pm 7.7$ vs $10.8 \pm 7.7 \mathrm{~mm}$, respectively; $P=.04$ ). patients in the platelet testing/PED group experienced ischemic stroke compared with $4(2.1 \%)$ patients in the PED alone group $(P=.06)$. The neurologic morbidity rate was higher in the platelet testing/PED group than in the PED alone group (42 [8.2\%] vs 4 [2.1\%] patients, respectively; $P=.01)$. There was also a trend toward a higher neurologic mortality rate in the platelet testing/ PED group than in the PED alone group (18 [3.5\%] vs 2 [1.1] patients, respectively; $P=.12$ ). The combined neurologic morbidity and mortality rate was higher in the platelet testing/PED group than in the PED alone group (45 [8.8\%] vs 6 [3.2\%] patients, respectively; $P=.01$ ). The all-cause mortality rate was also higher in the platelet testing/PED group than in the PED alone group. There were 3 cases of nonneurologic mortality, 2 in the platelet testing/PED group and 1 in the PED alone group. Causes of nonneurologic mortality included extracranial hemorrhage, sudden death, and hepatic fibrosis with cirrhosis. All adverse events occurred after PED placement. The median and mean times for complications were 7 and 40 days after the procedure, respectively (range, 0-397 days). These data are summarized in Table 2.

Multivariate Analysis. On multivariate logistic regression analysis with adjustment for age, aneurysm size, aneurysm location, number of PEDs, center volume, practitioner volume, and number of aneurysms, the platelet testing/PED group had a higher odds of neurologic morbidity (OR, 3.25 [95\% CI, 1.10-9.61]; $P=$ 
Table 3: Multivariate logistic regression analysis: odds of

\begin{tabular}{llcc}
\multicolumn{1}{c}{ Complications according to platelet-testing status } & \\
\hline \multicolumn{1}{c}{ Complication } & OR $^{\mathbf{a}}$ & $95 \% \mathrm{Cl}$ & $\boldsymbol{P}$ Value \\
\hline $\begin{array}{l}\text { Primary outcome: neurologic } \\
\text { morbidity and neurologic }\end{array}$ & 2.37 & 0.95 to 5.93 & .06 \\
$\begin{array}{l}\text { mortality } \\
\text { Secondary outcomes }\end{array}$ & & & \\
$\quad$ Spontaneous rupture & 1.46 & 0.28 to 7.67 & .65 \\
Intracranial hemorrhage & 6.56 & 0.50 to 85.80 & .15 \\
Ischemic stroke & 2.03 & 0.67 to 6.20 & .21 \\
Parent artery stenosis & 0.06 & $<0.001$ to 24.51 & .37 \\
Cranial neuropathy & 1.17 & 0.22 to 6.38 & .85 \\
Neurologic mortality & 3.24 & 0.80 to 15.01 & .13 \\
Neurologic morbidity & 3.25 & 1.10 to 9.61 & .03 \\
All-cause mortality & 3.67 & 0.81 to 16.74 & .09 \\
\hline
\end{tabular}

a Shown are the odds in the platelet testing/PED group versus those in the PED alone group. For each of the complications, the OR is for the platelet testing/PED versus the PED alone group. The analysis was adjusted for age, gender, aneurysm size, number of aneurysms treated, use of multiple PEDs, practitioner's previous experience, and center volume.

.03). There was also a trend in the platelet testing/PED group toward higher odds of combined neurologic morbidity and mortality (OR, 2.37 [95\% CI, 0.95-5.93]; $P=.064$ ) and all-cause mortality (OR, 3.67 [95\% CI, 0.81-16.74]; $P=.09$ ) relative to those in the PED alone group. For all the models, $P$ values from the Hosmer-Lemeshow goodness-of-fit test were nonsignificant $(P>.05)$, indicating that there was no evidence of poor fit. These data are summarized in Table 3.

\section{DISCUSSION}

Our study of patients included in the International Retrospective Study of Pipeline Embolization Device registry found higher complication rates and higher rates of neurologic morbidity in the platelet testing/PED group than in the PED alone group. However, there were significant differences in the baseline characteristics of the 2 groups. Namely, patients in the PED alone group were younger and were less likely to be treated with multiple PEDs, and the group had a larger mean aneurysm size. In addition, patients in the platelet testing/PED group were more likely to be treated at high-volume centers and to be treated by highervolume practitioners. In our analysis, which was adjusted for age, aneurysm size, aneurysm location, number of aneurysms, number of PEDs, center volume, and practitioner volume, platelet testing was associated with a significantly higher odds of neurologic morbidity. These findings are important, because they suggest that preoperative platelet testing does not result in improved outcomes of patients who undergo PED placement and is associated with higher odds of neurologic morbidity after PED treatment.

Previous studies have found variable associations between platelet response and ischemic and hemorrhagic complications after neurovascular stent placement and after PED placement. In a study of 96 patients who received a neurovascular (carotid or intracranial) stent, Fifi et $\mathrm{al}^{9}$ found that clopidogrel-resistant patients had a significantly higher rate of thromboembolic events than those who were not resistant. In a study of 74 patients who underwent PED placement, Tan et $\mathrm{al}^{12}$ found that having a level of $>208$ P2Y12 reaction units was associated with a non-statistically significant trend toward higher rates of symptomatic stroke on both univariate and multivariate analysis but no significant dif- ference in infarcts as seen in diffusion-weighted MR imaging. Delgado Almandoz et $\mathrm{al}^{13}$ found higher rates of stroke among patients with clopidogrel hyporesponse who underwent stentassisted coiling and PED placement and higher rates of hemorrhage among patients with clopidogrel hyperresponse. Similarly, Goh et al found that hyperresponse to clopidogrel was associated with higher rates of hemorrhage. ${ }^{14}$ Heller et al ${ }^{15}$ studied the effect of antiplatelet therapy on thromboembolic events after flow diversion with the PED and found no difference in platelet reactivity between patients with and without infarcts found with MR imaging.

Although previous studies found an association between platelet-testing results and neurovascular complications after stent/PED placement, studies have yet to clearly demonstrate that altering antiplatelet regimens on the basis of platelet-testing results leads to improved clinical outcomes. Fifi et $\mathrm{al}^{9}$ compared thromboembolic event rates among a group of patients who underwent changes in antiplatelet therapy regimens based on platelet-testing results and another group of patients who did not undergo any changes and found no difference in thromboembolic event rates. In a study that compared complication rates among patients who underwent platelet testing (68 patients) and those who did not (32 patients), Oran et $\mathrm{al}^{16}$ found higher rates of morbidity and thrombotic complications in the group that did not undergo testing $(P=.03)$. However, in a study of 81 patients who underwent flow-diverter placement, Nordeen et $\mathrm{al}^{17}$ found that despite the fact that most clopidogrel-resistant patients received higher loading and maintenance doses of clopidogrel, the mortality rate was significantly higher in the clopidogrel-resistant group, and higher loading doses were associated with a trend toward higher complication rates $(P=.07)$. The results of these studies range from demonstrating a mild benefit for platelet function testing to mild harm.

However, our study of $>800$ patients revealed that platelet function testing is associated with a significantly higher odds of morbidity in patients who undergo PED placement. Although it is unclear from this cohort exactly what the precise reasons might be for these findings, it is clear that testing by itself has no reason to affect outcome. We can speculate on the causes, but there are no available details in this dataset to indicate what actions were taken or not taken in response to the test results. We speculate that aggressive periprocedural manipulation of loading doses or other adjunctive drugs may be causal; however, there is no definitive evidence from this study to suggest that this is the case. It should be recognized, however, that there was a higher number of PEDs used in the platelet testing/PED group, which may have played a significant role in contributing to the morbidity related to the procedure. However, even when adjusting for this variable, the use of platelet testing was still associated with higher complication rates. Other unmeasured factors, such as the presence of comorbidities or baseline platelet dysfunction, may have contributed to the higher morbidity rate seen in the platelet testing/PED group. What may be recommended is that testing and any responses to the test results be performed days before the actual procedure to reduce the potential for overshooting targets and incurring potentially greater morbidity rates in the postprocedural period, as noted in this cohort of tested patients.

AJNR Am J Neuroradiol 36:2090-95 Nov 2015 www.ajnr.org 
The effects of altering antiplatelet therapy to alter the rate of adverse thrombotic events have been studied extensively in the cardiology literature. The Gauging Responsiveness with a VerifyNow Assay-Impact on Thrombosis and Safety (GRAVITAS) trial compared the effects of high-dose and standard-dose clopidogrel with high on-treatment platelet reactivity after percutaneous coronary intervention; no differences in fatal cardiovascular events, stent thrombosis, or nonfatal myocardial infarction were seen between the standard- and high-dose-clopidogrel groups, ${ }^{18}$ despite the fact that the high-dose group had significantly decreased platelet activity compared with that in the standard-dose group. Although the GRAVITAS study did not find a benefit with increased clopidogrel doses, it demonstrated an increased incidence of adverse events in the high-reactivity group compared with that of the clopidogrel responders. A meta-analysis of 10 randomized clinical trials in which an intensified antiplatelet protocol based on adenosine diphosphate-specific platelet-reactivity testing was used found that the intensified protocol was associated with lower mortality and myocardial infarction rates; however, the benefit of intensified antiplatelet therapy was highly dependent on the patient's initial risk of stent thrombosis. ${ }^{19}$ In a recent position paper by the Working Group of the European Society of Cardiology, high platelet reactivity (resistance to clopidogrel) was recognized as a marker for a high event rate during coronary intervention, especially during acute myocardial infarct intervention. ${ }^{20}$ The use of alternative second antiplatelet agents such as prasugrel and ticagrelor has been recommended in such cases. The significance and translation of these findings to neurointervention remain unclear.

\section{Limitations}

Our study had limitations. It was a retrospective study in which sites followed their own standard of practice for treating aneurysms with PED placement, and there was a wide range of treatment regimens (eg, antiplatelet therapy) between centers. Although we compared complication rates in the platelet testing/ PED and PED alone groups, we do not have information regarding which patients in the testing group were nonresponders or hyperresponders. Thus, it is possible that more patients in the PED alone group had normal platelet function. We cannot report data on complication rates by antiplatelet-response status. We included only patients with an unruptured aneurysm, so the results of this study may not apply to patients with a ruptured aneurysm. Furthermore, we do not have information regarding which patients had changes to their antiplatelet regimens based on results from platelet-response testing. Last, outcomes were not ascertained while blinded to testing status. Overall, however, our study shows that platelet testing in and of itself does not lead to superior outcomes among patients who undergo PED placement.

\section{CONCLUSIONS}

Our study from a large retrospective registry shows that platelet testing of patients who underwent PED placement was not associated with improved outcomes. The underlying cause of this finding is unclear. Further prospective studies, similar to those published in the cardiology literature, are needed to determine if and when platelet testing and correction of antiplatelet therapies are appropriate in patients who undergo PED placement.

Disclosures: Giuseppe Lanzino-UNRELATED: Consultancy: ev3/Covidien.* Adnan Siddiqui_RELATED: Consulting Fee or Honorarium: Covidien, Comments: Consulting fees for Pipeline Proctoring and Scientific Advisory Board Membership, Member of the Covidien SWIFT PRIME National Steering Committee; UNRELATED: Board Membership: Codman and Shurtleff, ICAVL, and Medina Medical; Consultancy: Codman \& Shurtleff, Guidepoint Global, Peneumbra, Stryker, Pulsar Vascular, MicroVention, Lazarus Effect, Blockade Medical, Reverse Medical, and Abbott Vascular; Payment for Lectures (including service on speakers bureaus): Codman \& Shurtleff; Stock/Stock Options: Hotspur, Intratech Medical, StimSox, Valor Medical, Blockade Medical, Lazarus Effect, Pulsar Vascular, and Medina Medical; Other: Penumbra and MicroVention, Comments: Member of Penumbra 3D Separator Trial National Steering Committee, Member of MicroVention FRED Trial National Steering Committee. Ricardo Hanel-RELATED: Consulting Fee or Honorarium: Covidien, Stryker, and Codman; UNRELATED: Board Membership: Medina; Stock/Stock Options: Blockade. David F. Kallmes—RELATED: Grant: ev3/Covidien, ${ }^{*}$ Comments: Clinical trial implementation; Consulting Fee or Honorarium: ev3/Covidien, ${ }^{*}$ Comments: Safety Monitoring Board and Steering Committee participation; UNRELATED: Board Membership: GE Healthcare, Comments: Cost-effectiveness board; Consultancy: ev3/ Covidien,* Comments: Planning and implementing clinical trials; Grants/Grants Pending: MicroVention, ${ }^{*}$ Codman, ${ }^{*}$ SurModics, ${ }^{*}$ Sequent, ${ }^{*}$ NeuroSigma, ${ }^{*}$ and ev3, ${ }^{*}$ Comments: Preclinical and clinical research. *Money paid to institution.

\section{REFERENCES}

1. Arrese I, Sarabia R, Pintado R, et al. Flow-diverter devices for intracranial aneurysms: systematic review and meta-analysis. Neurosurgery 2013;73:193-99; discussion 199-200 CrossRef Medline

2. Briganti F, Napoli M, Tortora F, et al. Italian multicenter experience with flow-diverter devices for intracranial unruptured aneurysm treatment with periprocedural complications-a retrospective data analysis. Neuroradiology 2012;54:1145-52 CrossRef Medline

3. Brinjikji W, Murad MH, Lanzino G, et al. Endovascular treatment of intracranial aneurysms with flow diverters: a meta-analysis. Stroke 2013;44:442-47 CrossRef Medline

4. Yu SC, Kwok CK, Cheng PW, et al. Intracranial aneurysms: midterm outcome of Pipeline embolization device-a prospective study in $\mathbf{1 4 3}$ patients with $\mathbf{1 7 8}$ aneurysms. Radiology 2012;265:893901 CrossRef Medline

5. Kallmes DF, Ding YH, Dai D, et al. A new endoluminal, flow-disrupting device for treatment of saccular aneurysms. Stroke 2007;38: 2346-52 CrossRef Medline

6. Kallmes DF, Ding YH, Dai D, et al. A second-generation, endoluminal, flow-disrupting device for treatment of saccular aneurysms. AJNR Am J Neuroradiol 2009;30:1153-58 CrossRef Medline

7. Karaźniewicz-Lada M, Danielak D, Burchardt P, et al. Clinical pharmacokinetics of clopidogrel and its metabolites in patients with cardiovascular diseases. Clin Pharmacokinet 2014;53:155-64 CrossRef Medline

8. Delgado Almandoz JE, Crandall BM, Scholz JM, et al. Pre-procedure P2Y12 reaction units value predicts perioperative thromboembolic and hemorrhagic complications in patients with cerebral aneurysms treated with the Pipeline embolization device. J Neurointerv Surg 2013;5(suppl 3):iii3-10 CrossRef Medline

9. Fifi JT, Brockington C, Narang J, et al. Clopidogrel resistance is associated with thromboembolic complications in patients undergoing neurovascular stenting. AJNR Am J Neuroradiol 2013;34:716-20 CrossRef Medline

10. Comin J, Kallmes DF. Platelet-function testing in patients undergoing neurovascular procedures: caught between a rock and a hard place. AJNR Am J Neuroradiol 2013;34:730-34 CrossRef Medline

11. Kallmes DF, Hanel R, Lopes D, et al. International retrospective study of the Pipeline embolization device: a multicenter aneurysm treatment study. AJNR Am J Neuroradiol 2015;36:108-15 CrossRef Medline

12. Tan LA, Keigher KM, Munich SA, et al. Thromboembolic complications with Pipeline embolization device placement: impact of procedure time, number of stents and pre-procedure $\mathrm{P} 2 \mathrm{Y} 12$ reaction 
unit (PRU) value. J Neurointerv Surg 2015;7:217-21 CrossRef Medline

13. Delgado Almandoz JE, Kadkhodayan Y, Crandall BM, et al. Variability in initial response to standard clopidogrel therapy, delayed conversion to clopidogrel hyper-response, and associated thromboembolic and hemorrhagic complications in patients undergoing endovascular treatment of unruptured cerebral aneurysms. J Neurointerv Surg 2014;6:767-73 CrossRef Medline

14. Goh C, Churilov L, Mitchell P, et al. Clopidogrel hyper-response and bleeding risk in neurointerventional procedures. AJNR Am J Neuroradiol 2013;34:721-26 CrossRef Medline

15. Heller RS, Dandamudi V, Lanfranchi M, et al. Effect of antiplatelet therapy on thromboembolism after flow diversion with the Pipeline embolization device. J Neurosurg 2013;119:1603-10 CrossRef Medline

16. Oran I, Cinar C, Bozkaya H, et al. Tailoring platelet inhibition according to multiple electrode aggregometry decreases the rate of thrombotic complications after intracranial flow-diverting stent implantation. J Neurointerv Surg 2015;7:357-62 CrossRef Medline
17. Nordeen JD, Patel AV, Darracott RM, et al. Clopidogrel resistance by P2Y12 platelet function testing in patients undergoing neuroendovascular procedures: incidence of ischemic and hemorrhagic complications. J Vasc Interv Neurol 2013;6:26-34 Medline

18. Price MJ, Berger PB, Teirstein PS, et al; GRAVITAS Investigators. Standard- vs high-dose clopidogrel based on platelet function testing after percutaneous coronary intervention: the GRAVITAS randomized trial. JAMA 2011;305:1097-105 CrossRef Medline

19. Aradi D, Komócsi A, Price MJ, et al; Tailored Antiplatelet Treatment Study Collaboration. Efficacy and safety of intensified antiplatelet therapy on the basis of platelet reactivity testing in patients after percutaneous coronary intervention: systematic review and metaanalysis. Int J Cardiol 2013;167:2140 - 48 CrossRef Medline

20. Aradi D, Storey RF, Komócsi A, et al; Working Group on Thrombosis of the European Society of Cardiology. Expert position paper on the role of platelet function testing in patients undergoing percutaneous coronary intervention. Eur Heart J 2014;35:209-15 CrossRef Medline 